\title{
Research on the Application of Object-Oriented Software Engineering Ying Ding ${ }^{1,}$ a Xiaodong Zhang ${ }^{2, b}$ \\ ${ }^{1}$ Zhengzhou Institute of Technology, Zhengzhou, Henan, China, 450044 \\ ${ }^{2}$ Henan Mechanical and Electrical Vocational College, Zhengzhou, Henan, China, 451191 \\ ${ }^{a}$ email, ${ }^{b}$ email,
}

Keywords: Research, Application, Object-Oriented Software Engineering

\begin{abstract}
Object-oriented software engineering is a hot object in the field of software engineering research and development. Based on the software engineering methodology and the development of application software system, this paper studies the problems in object-oriented software engineering research and development. And then discusses several commonly methods used in object-oriented software engineering development, discusses the characteristics, basic functions and development trends of object-oriented software engineering.
\end{abstract}

\section{Introduction}

In recent years, with the progress of science and technology and the development of the times, object-oriented software engineering is also more and more attention. Software development methods can use the existing symbols to organize the production of software and the purpose is in the prescribed human and financial resources and time to develop the software to meet the requirements. Object-oriented software engineering refers to the object-oriented, at the same time in a way that people can easily understand the way to face the problems in reality and develop the corresponding software to be addressed, it is the object-oriented approach in the field of software engineering Perfect combination and full use of software engineering research has brought a new way of thinking and methods, the field of software engineering is a rare good tool. Object-oriented software engineering methods are formed step by step, from the face to face programming languages to reflect the basic ideas to integrate in the analysis and design methods, in the past few decades, the face of software engineering methods have been rapid development. Today's object-oriented software engineering methods include the main content of the problem domain, natural language, object-oriented editing language and so on. In this paper, based on the basis of previous research, the commonly used object-oriented software engineering methods were discussed.

\section{The Definition of Software Engineering}

Software engineering is to guide the development of computer software and maintenance engineering disciplines. Develop and maintain software using the concepts, principles, techniques, and methods of engineering, combine time-tested and proven management techniques with the best available technologies today, economically develop high-quality software and efficiently Maintain it, this is the software engineering.

Software engineering using computer science, mathematics and management science and other principles, learn from traditional engineering principles, methods, to create software to improve quality and reduce costs. Among them, computer science, mathematics for the construction of models and algorithms, engineering science for manufacturing norms, assess the cost and determine the trade-offs, management science for planning, resource quality, cost management.

\section{The Process of Software Engineering Methods}

Software Planning. In the design task before the establishment of the first to conduct research and feasibility studies, understand the scope of work and the cost, and then make a software plan. 
Software Requirements Analysis. The user requirements for a specific analysis to determine the user requirements of the software system to do what, and software requirements specification expressed as a user and software personnel between the common agreements.

Software Design. Describe the structure of the software system, including the data structure and the module structure. This part is divided into the overall design and detailed design of two stages.

Software Coding. Write the program for each module as required by the software design.

Software Testing. Find and eliminate errors in the program, tested and debugged, get the software to run. Software testing is divided into unit testing and comprehensive testing of two stages.

Software Maintenance. The software that is tested may still be in error. In addition, the user's requirements and the operating environment of the system may change. Therefore, the software that is delivered still needs to be debugged, modified, and expanded. This is software maintenance.

\section{The Object-Oriented Analysis}

Object Oriented Analysis (OOA) is a technology widely used by software production and development enterprises. OOA is composed of five major steps: identifying objects, identifying structures, defining themes, defining attributes (and instance connections) and defining services (and message connections). . This approach is still object-centric, requiring the problem domain to be classified by class or object, etc., and then to identify the characteristics of these objects and the relationship between each other, thereby creating a unified specification to solve the problem of a detailed description.

The Advantages of Object-Oriented Analysis. As one of the most important technologies of modern software production and development, object-oriented analysis must have its advantage of irreplaceable and imitating. The following will enumerate a few: First, object-oriented analysis of the problem areas of focus on understanding and understanding, help solve the problem; Second, it is flexible and convenient in the current actual operation of the shunt and analysis of the differences between the adjustment It uses the self-contained sub-block which has the least dependency among the objects to explain and analyze, so that it can be used as a unified whole.

The Specific Operation Process of Object-Oriented Analysis. First, the drawing generated use case diagram. A use case is a sequence of things that can be done in a system session in relation to the behavior of the user using the system. There are three relationships between use cases: the connection between roles and use cases, the use and extension between use cases, and the use of roles to identify non-use cases; Second, the construction of the static system model. Static models, including class diagrams, object graphs, etc., reflect the types of objects in the software system and the static relationships between them. It goes without saying that static models are also constructed with objects as the center. These are class diagrams, and so on; Thirdly, draw the dynamic model of building systems. The process of development of things always contains dynamic and static processes, static and dynamic combination of the two complements each other, inseparable. Similarly, the object-oriented analysis of static models, there is a dynamic model, it is the implementation of various insurance system sequence state and the relationship between the performances of the system behavior. The dynamic model is a description of the system activity and the dynamic cooperation relationship, etc., relative to the description of the characteristic types of the various objects in the static model.

\section{The Object-Oriented Design}

Object-oriented design is independent of the programming language to describe the software design, is also flexible and convenient. It is based on the thorough understanding and understanding of "object-oriented". It solves the organizational relationship between classes and objects that communicate with each other. It has the same concept, principle and representation method as object-oriented analysis. Object-oriented design of the specific process is as follows:

(1) The further refinements of the object behavior and object interaction. (2) The design and 
implementation of use cases. In the object-oriented analysis to generate use-case diagram of the operations and steps so that we have a certain understanding of the use case, but it is only from the outside of the system to show the function of the system is not comprehensive. As a perfect and complementary, object-oriented design in the use case design to achieve the system's internal objects and external functions of the interaction and adaptation. (3) Detailed analysis of the interaction phase diagram, state diagram, activity diagram. (4) To determine the type of system reusable components or components. (5) Using the component diagram to describe the physical structure of the code components and the dependencies between each other. (6) With the configuration diagram describes the physical and software structure of the hardware and software. Object-oriented analysis is a direct observation of the problem areas, analysis, so that the system can directly map out the problem areas, to maintain the integrity of the object in the problem area and authenticity, and object-oriented design is object-oriented design object model made Abstract and inductive, to generate intuitive inductive graphics, the object model to be generalized and sublimation. From this point of view, object-oriented design is the data refinement and abstraction process, is the object-oriented software engineering methods important tools and methods.

\section{The Object-Oriented Software Engineering Methods}

The Definition of Object-Oriented Software Engineering Method. Object-oriented technology is a very practical and powerful development method. It is a kind of software tool created to improve the stability, modifiability and reusability of the software system. It has the characteristics of uniqueness and high continuity of the method, and combines object-oriented analysis, object-oriented design and object-oriented programming Integrated into the corresponding phase of the software life cycle. Object-oriented software engineering from top to bottom, from beginning to end to implement the object-oriented ideas, can be used for system analysis, system design, programming, can also be used for other environmental development, such as data structure design, database design.

The Features and Key Points of Object-Oriented Software Engineering Methods. Object-oriented software engineering approach to the starting point and the basic principle is to simulate human habit of thinking as much as possible, so that the development of software methods and processes as close as possible to human understanding of the world to solve the problem of the method and process, which is to describe the problem space and Therefore, the object-oriented approach has the following basic characteristics: (1) Encapsulation: the object is the data and the operation of the package body. (2) Inheritance and analogy: In the object inheritance structure, the lower object inherits the characteristics of the upper object properties and operations. When different objects have some of the same attributes, they are often grouped together. (3) Dynamic connectivity: between objects and objects with a unified, convenient, dynamic connection and the ability to transfer messages and mechanisms. Dynamic connectivity facilitates conceptual parallel and distributed architectures. (4) Easy to maintain: any object is how to achieve the details of the object function hidden in the internal object, which enhances the object and the entire system easier to maintain.

Broadly speaking, object-oriented approach has the following four points: (1) the object as the integration of data and data on the operating behavior of the unified software components. Object-oriented programs are composed of objects, any element in the program is an object, complex objects from a relatively simple combination of objects. (2) All objects are divided into classes. Each class defines a set of data and a set of operations, the class is the same data and the same operation on a group of similar objects definition .Data used to represent the object (3) In accordance with the relationship between the parent class and the sub-class, a number of related classes into a hierarchical structure of the system. In the class (class), the class of the class, (4) objects can only be sent to each other by sending messages to each other. Objects are essentially different from traditional data, it is the nature of the difference between the traditional data, it is not the same as the traditional data, Instead of waiting for the outside world to act on it, it is the subject of processing and has to send it a message asking it to do something with it to process its data 
instead of processing it directly from the outside world.

The Object-Oriented Modeling. In order to better understand the problem, it is often used to establish the problem model approach. Often, a model consists of a set of graphic symbols and rules that organize the symbols, which are used to define and describe the terms and concepts in the problem domain. Furthermore, the model is a thinking tool, the use of this tool can be standardized knowledge that.

Object-oriented method development of software, usually need to establish three forms of models, which are described in the data structure of the object model, describe the dynamic control system structure and describe the functional model of the functional model. These three models share common concepts of data, control, and operations, with each model describing different emphases. These three models model the target system from three different but closely related perspectives, each of which Reflecting the substantive content of the system, together it is a comprehensive reflection of the target system needs.

\section{Conclusion}

In summary, the object-oriented software engineering approach will use the object-oriented ideas in the software development process. Its main idea is established on the basic concept of the object. Although the object-oriented software engineering method has some shortcomings, it has more advantages compared to traditional software engineering methods. The object-oriented software engineering methods highlights the logic thinking and changed the too mechanized shortcomings of the traditional software engineering method, it is more realistic and specific, more conducive to people's understanding of software engineering methods. It improves the efficiency and effectiveness of software production and provides a new way and thinking of software development.

\section{References}

[1] Jia Xinzhang, Li Jingyuan. Shangrao Normal University, Vol. 6 (2014) No 53, p.25-26

[2] Wang Qunyong. Development of Microcomputer, Vol. 12 (2015) No 27, p.74-76

[3] Qian Xiyuan, Jing Jianfen. Information and Computers, Vol. 30 (2014) No 19, p.144-145

[4] Wang Li. Computer CD - ROM Software and Application, Vol. 29 (2008) No 27, p.21-23

[5] Zhang Gongxu, Sun Jing. Modern Commerce and Industry, Vol. 8 (2013) No 27, p.57-60 